\title{
Disulfiram Induced Psychosis
}

\author{
Satyakam Mohapatra, Nihar Ranjan Rath \\ Mental Health Institute, S.C.B. Medical College, Cuttack, India
}

Disulfiram is the commonly prescribed drug for the treatment of alcohol dependence. It's major metabolite (diethyldithiocarbamate) is an inhibitor of dopamine-betahydroxylase, an enzyme that catalyzes the metabolism of dopamine to norepinephrine resulting in psychosis. We recommend that disulfiram should be used at the lowest effective dose, possibly 250 mg daily and caution should be taken while prescribing disulfiram for patients with personal and familial antecedents of psychosis.

KEY WORDS: Disulfiram; Psychosis; Side effect.

\section{INTRODUCTION}

For the past 50 years disulfiram is the commonly prescribed drug for the treatment of alcohol dependence. Important side effects of disulfiram are hepatological, dermatological, neurological (polyneuritis, encephalopathy) ${ }^{1,2)}$ and psychiatric in nature. Psychiatric manifestations include confusion, loss of memory, psychosis, ${ }^{3-6)}$ mania with psychotic symptoms. ${ }^{7,8)}$ Disulfiram's major metabolite diethyldithiocarbamate is an inhibitor of dopaminebetahydroxylase (DBH), an enzyme that catalyzes the metabolism of dopamine (DA) to norepinephrine (NE). ${ }^{9}$ By inhibiting this metabolic pathway from DA to NE in the central nervous system, disulfiram results in an increase of DA concentrations in mesolimbic system resulting in psychosis. Alcoholics who developed psychotic symptoms during disulfiram treatment are found to have low levels of amine and monoamine oxidase, suggesting DBH blockage. ${ }^{10)}$ We present a case of 32-year-old male who developed psychosis with the use of disulfiram.

\section{CASE}

A 32-year-old male without past of psychiatric and neurological illness reported to psychiatry outpatient department with complaint of daily alcohol intake of $250-750 \mathrm{ml}$

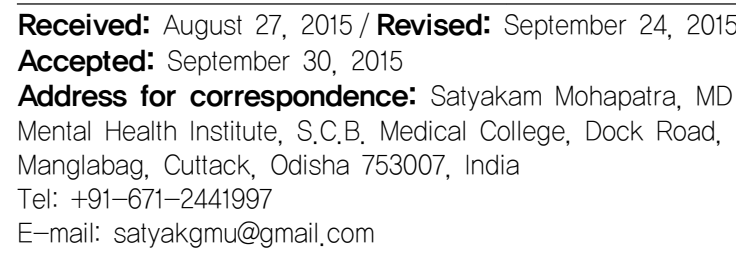

( $40 \%$ alcohol by volume) for the last 5 years. With the informed consent of the patient, disulfiram was administered at the dosage of $250 \mathrm{mg}$ twice daily for 1 month, while the patient abstained from alcohol. Then the patient presented complaint of fearfulness, suspiciousness, easy irritability, muttering to self, decreased sleep for last one month. Detailed medical evaluation revealed no significant findings. Haematological and biochemical indices were within normal limits. On mental status examination delusion of persecution and auditory hallucinations were elicited. He has family history of schizophrenia and comorbid alcohol dependence in his father. The patient has no history of any other substance abuse. The patient was hospitalised and possibility of psychosis induced by disulfiram was kept. Disulfiram was stopped. Symptoms of alcohol withdrawal and disulfiram-alcohol reactions were not observed during hospitalization. He was started on tablet lorazepam $4 \mathrm{mg}$ per day. His fearfulness, suspiciousness, irritability, muttering to self and impaired sleep gradually improved over a period of 10 days. After 2 weeks he improved completely and lorazepam was stopped.

\section{DISCUSSION}

Risk factors for development of disulfiram related psychotic symptoms include past history or family history of psychosis, overly rapid increase in dosage or greater than recommended total dosage, old age, impaired liver function and concurrent dopaminergic medications or psycho-stimulant abuse. ${ }^{5,11)}$ Our patient has family history of schizophrenia in his father. His psychotic symptoms im-

(c) This is an Open-Access article distributed under the terms of the Creative Commons Attribution Non-Commercial License (http://creativecommons.org/licenses/by-nc/4.0) which permits unrestricted non-commercial use, distribution, and reproduction in any medium, provided the original work is properly cited. 
proved after stoppage of disulfiram and use of lorazepam tablet only without any additional requirement of antipsychotic treatment. These factors support the association between with disulfiram use and emergence of psychotic symptoms in our patient.

The usual recommended initial dosage is $500 \mathrm{mg}$ per day for the first 1 or 2 weeks, followed by a maintenance dosage of $250 \mathrm{mg}$ per day. ${ }^{12)}$ Disulfiram related psychosis usually involve either therapeutic or higher than recommended dosages. ${ }^{13)}$ Our patient has developed psychotic symptoms with $500 \mathrm{mg}$ per day of disulfiram. We recommend that disulfiram should be used at the lowest effective dose, possibly $250 \mathrm{mg}$ daily. Our case highlights the relevance of a careful history of patient's symptoms and family history that should be performed before starting treatment with disulfiram and caution should be taken while prescribing disulfiram for patients with personal and familial antecedents of psychosis. Disulfiram-related psychiatric complications are reported to be more prevalent in eastern countries, ${ }^{3)}$ which suggests that genetic factors may play a role in disulfiram induced psychosis.

\section{REFERENCES}

1. Mohapatra S, Sahoo MR, Rath N. Disulfiram-induced neuropathy: a case report. Gen Hosp Psychiatry 2015;37:97.e5-e6.
2. Hotson JR, Langston JW. Disulfiram-induced encephalopathy. Arch Neurol 1976;33:141-142.

3. Murthy KK. Psychosis during disulfiram therapy for alcoholism. J Indian Med Assoc 1997;95:80-81.

4. Quail M, Karelse RH. Disulfiram psychosis. A case report. $S$ Afr Med J 1980;57:551-552.

5. Rex HH, Møller-Madsen S. Psychosis due to an overdose of disulfiram (Antabus). Ugeskr Laeger 1986;148:1679-1680.

6. Krishna Murthy K, Praveenlal K. An experience with disulfiram in the management of alcohol dependence syndrome. Indian J Psychol Med 1988;11:145-148.

7. Ceylan ME, Turkcan A, Mutlu E, Onal O. Manic episode with psychotic symptoms associated with high dose of disulfiram: a case report. J Clin Psychopharmacol 2007;27: 224-225.

8. Li MY, Shen YC. Manic episode with psychosis following a lower than recommended dosage regimen of disulfiram. Prog Neuropsychopharmacol Biol Psychiatry 2008;32:311-312.

9. Murphy DL, Goodwin FK, Brodie HK, Bunney WE Jr. L-dopa, dopamine, and hypomania. Am J Psychiatry 1973; 130:79-82.

10. Nasrallah HA. Vulnerability to disulfiram psychosis. West $J$ Med 1979;130:575-577.

11. Mackie J, Clark D. Cannabis toxic psychosis while on disulfiram. Br J Psychiatry 1994;164:421.

12. Brewer C. How effective is the standard dose of disulfiram? A review of the alcohol-disulfiram reaction in practice. $\mathrm{Br}$ J Psychiatry 1984;144:200-202.

13. Larson EW, Olincy A, Rummans TA, Morse RM. Disulfiram treatment of patients with both alcohol dependence and other psychiatric disorders: a review. Alcohol Clin Exp Res 1992;16:125-130. 